\title{
External Quality Assessment Evaluating the Ability of Dutch Clinical Microbiological Laboratories to Identify Candida auris
}

\author{
Jochem B. Buil ${ }^{1,2, *(D)}$, Henrich A. L. van der Lee ${ }^{1,2}$, Ilse Curfs-Breuker ${ }^{1,2,3}$, Paul E. Verweij ${ }^{1,2}$ \\ and Jacques F. Meis $1,2,3$ (D) \\ 1 Department of Medical Microbiology, Radboud University Medical Center, 6525GA Nijmegen, \\ The Netherlands; Henrich.vanderLee@radboudumc.nl (H.A.L.v.d.L.); \\ Ilse.Curfs-Breuker@radboudumc.nl (I.C.-B.); paul.verweij@radboudumc.nl (P.E.V.); \\ jacques.meis@gmail.com (J.F.M.) \\ 2 Center of Expertise in Mycology Radboudumc/CWZ, 6525GA Nijmegen, The Netherlands \\ 3 Department of Medical Microbiology and Infectious Diseases, Canisius-Wilhelmina Hospital (CWZ), \\ 6532SZ Nijmegen, The Netherlands \\ * Correspondence: jochem.buil@radboudumc.nl; Tel.: +31-24361-4356
}

Received: 30 August 2019; Accepted: 30 September 2019; Published: 7 October 2019

\begin{abstract}
Background: Candida auris is a yeast that is causing nosocomial outbreaks in healthcare facilities around the world. There is a risk of the misidentification of C. auris with matrix-assisted laser desorption ionization-time of flight mass spectrometry (MALDI-TOF MS)—when libraries are used that lack C. auris spectra, or when conventional biochemical methods are used. Methods: We conducted an external quality assessment to evaluate the ability of Dutch clinical microbiological laboratories to identify C. auris, and to raise awareness about the risk of misidentification. Results: 35/47 participating laboratories were able to identify C. auris correctly. Only 2/14 labs that potentially misidentified $C$. auris with their primary identification methods specified that they would perform additional tests to exclude C. auris when appropriate. 45/47 labs used MALDI-TOF MS systems to identify Candida species. Conclusions: There was a lack of awareness about the potential misidentification of $C$. auris in many labs that used MALDI-TOF MS with libraries that lacked $C$. auris spectra, and labs that used Vitek 2. However, as the currently available MALDI-TOF MS libraries in The Netherlands contain several C. auris spectra, we expect that currently almost all participating laboratories are able to identify C. auris correctly, as 45/47 participating laboratories use MALDI-TOF MS as their primary yeast identification method.
\end{abstract}

Keywords: C. auris; MALDI-TOF MS; misidentification

\section{Introduction}

Candida auris is an ascomycete yeast that is causing nosocomial outbreaks in healthcare settings around the world [1-17]. The yeast is an emerging pathogen and is considered a serious global health threat, due to its common multidrug-resistant phenotype and ability to cause outbreaks in health care settings [18-21]. The lack of its early correct identification, and the failure to implement the immediate contact precautions of infected or colonized patients, are the key conditions in need of consideration to prevent hospital transmissions and silent outbreaks. Reliable identification of C. auris is possible by sequence analysis of the D1/D2 region of the large subunit and the internal transcribed region of the ribosomal RNA, or by matrix-assisted laser desorption ionization-time of flight mass spectrometry (MALDI-TOF MS) [22-26]. However, there is a risk of misidentification when biochemical assays are used, or when the reference libraries lack C. auris spectra in their databases when MALDI-TOF MS 
systems are used. We organized an external quality assessment (EQA), to evaluate the ability of Dutch clinical microbiology laboratories to correctly identify $C$. auris isolates, and to raise awareness about this pathogen's potential to cause hospital outbreaks, and also the possibility of misidentifications.

\section{Materials and Methods}

The EQA panel consisted of six samples: Three samples containing C. auris isolates (V238-35, V238-38, and V238-40), two with C. haemulonii species complex (V238-33, containing both C. haemulonii/pseudohaemulonii and V238-39 C. duobushaemulonii), and V238-37 Candida lusitaniae. The isolates were identified by sequence analysis of the internal transcribed spacer region 1 and 2 [24]. Isolates were grown on Sabouraud dextrose agar plates and transferred to Sabouraud dextrose agar slants in a screw-cab tube, and incubated for $24 \mathrm{~h}$ at $35^{\circ} \mathrm{C}$. Then, in February 2018, the agar slants were sent to 63 clinical microbiology laboratories in The Netherlands. A questionnaire was added, containing a series of questions about the available methods for the identification of yeasts, and on which isolates these methods would be used. Laboratories were asked to report their results by returning the questionnaire and identification results by mail. The Center of Expertise in Mycology Radboudumc/CWZ analyzed the results, and returned the conclusions anonymously to all participants.

\section{Results}

\section{Identification}

Seven university hospital laboratories and 40 teaching and non-teaching hospital laboratories took part in the EQA. Thirty-five of $47(74 \%)$ laboratories correctly identified the three $C$. auris isolates. The $C$. auris isolate V238-35 was wrongly reported as $C$. haemulonii by $4(9 \%)$ laboratories, as $C$. famata and C. pelliculosa by one (2\%) laboratory, and as Candida species or yeast by $6(13 \%)$ laboratories. C. auris isolate V238-38 was misidentified as C. haemulonii by $5(11 \%)$ laboratories, and as Candida species or yeast by $7(15 \%)$ laboratories. C. auris isolate V238-40 was misidentified as $C$. haemulonii in $6(13 \%)$ laboratories, and as Candida species or yeast by $6(13 \%)$ labs. The mix of C. haemulonii and C. pseudohaemulonii C238-33 was identified as either C. haemulonii or C. pseudohaemulonii by $44(94 \%)$ laboratories, and as Candida species by $3(6 \%)$ laboratories. C. duobushaemulonii isolate V238-39 was correctly identified by $31(66 \%)$ laboratories, and wrongly as C. haemulonii by $16(34 \%)$. All laboratories correctly identified C. lusitaniae isolate V238-37 (Figure 1). Thirty-two of 33 (97\%) laboratories that used the Bruker MALDI Biotyper MALDI-TOF MS system (Bruker daltonics, Bremen, Germany) correctly identified the three $C$. auris isolates; while this was the case for three of $12(25 \%)$ laboratories that used the bioMérieux Vitek MS MALDI-TOF MS system (bioMérieux. Vitek MS, Marcy-l'Étoile, France) (Table 1). However, three laboratories that used the Vitek MS system indicated that additional methods were used to identify C. auris; two labs performed ITS sequencing and one lab used qPCR. Another lab indicated that they would send unidentified Candida isolates to a reference laboratory for identification.

Table 1. Identification of C. auris, C. haemulonii complex, and C. lusitatiae by two MALDI-TOF MS systems.

\begin{tabular}{ccc}
\hline Isolate & Bruker MALDI Biotyper & bioMérieux. Vitek MS \\
\hline C. haemulonii complex & $32 / 33$ & $10 / 12$ \\
C. auris & $32 / 33$ & $3 / 12^{1}$ \\
C. lusitaniae & $33 / 33$ & $12 / 12$ \\
C. auris & $32 / 33$ & $3 / 12^{1}$ \\
C. haemulonii complex & $33 / 33$ & $12 / 12$ \\
C. auris & $32 / 33$ & $3 / 12^{1}$ \\
\hline
\end{tabular}

${ }^{1}$ Identified as $C$. auris with additional methods. 
Six out of seven $(86 \%)$ clinical microbiology laboratories of university medical centers correctly identified the three $C$. auris strains, while 30 of $40(75 \%)$ teaching and non-teaching hospitals correctly identified the isolates (Table 2).

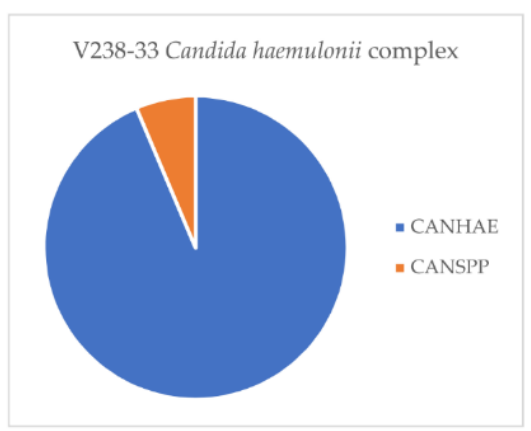

(a)

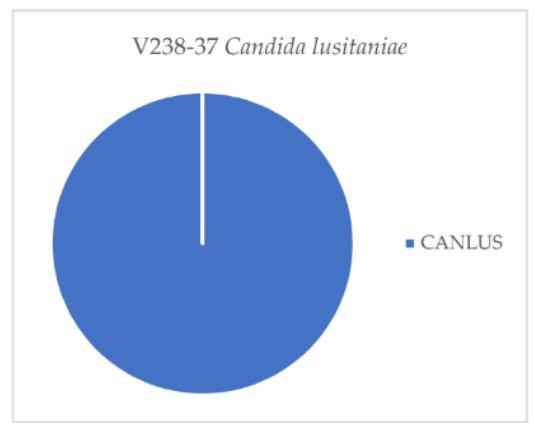

(c)

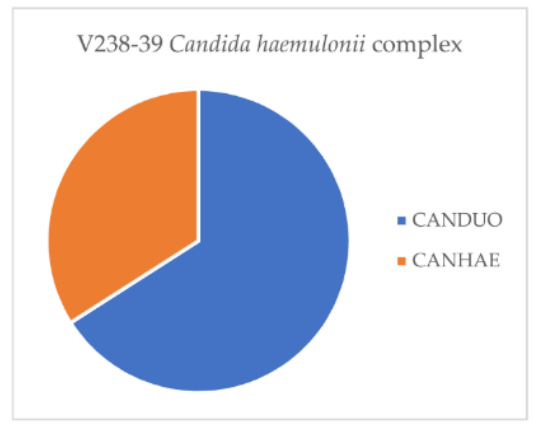

(e)

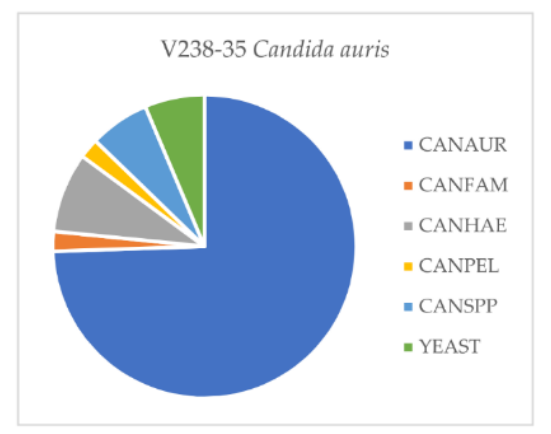

(b)

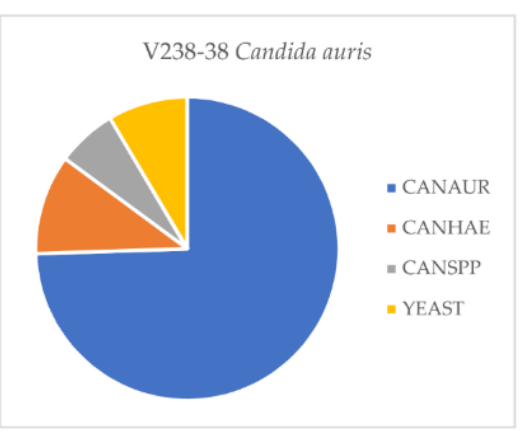

(d)

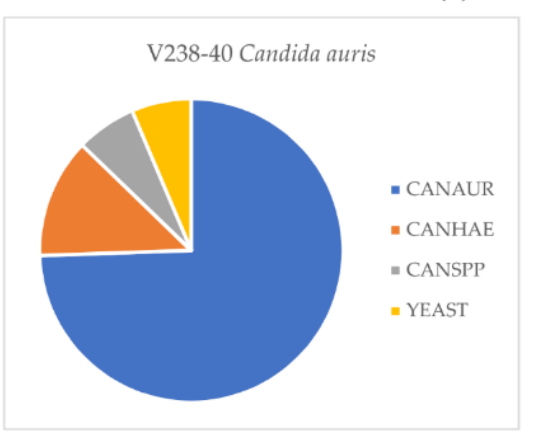

(f)

Figure 1. Identifications provided by 46 Dutch clinical microbiology laboratories for a panel of six Candida isolates. (a) C. haemulonii complex (containing both C. haemulonii and C. pseudohaemulonii); (b) C. auris; (c) C. lusitaniae; (d) C. auris; (e) C. haemulonii complex (C. duobushaemulonii); and (f) C. auris. CANAUR: C. auris, CANDUO: C. duobushaemulonii, CANFAM: C. famata, CANHAE: C. haemulonii, CANPEL: C. pelliculosa, CANLUS: C. lusitaniae, CANSPP: Candida species.

Table 2. Identification of C. auris, C. haemulonii complex, and C. lusitatiae by university and community hospitals.

\begin{tabular}{ccc}
\hline Isolate & University Hospital & Community Hospitals \\
\hline Chaemulonii complex & $6 / 7$ & $38 / 40$ \\
C. auris & $6 / 7$ & $30 / 40$ \\
C. lusitaniae & $7 / 7$ & $40 / 40$ \\
C. auris & $6 / 7$ & $30 / 40$ \\
Chaemulonii complex & $7 / 7$ & $40 / 40$ \\
C. auris & $6 / 7$ & $30 / 40$ \\
\hline
\end{tabular}


The two laboratories that did not use MALDI-TOF MS for the identification of yeast were not able to identify the $C$. auris isolates with their available methods. Both laboratories indicated that the unidentified isolates would have been sent to another laboratory for identification. However, both used Vitek 2 for identification, and this method identified the $C$. auris isolates as $C$. haemulonii.

\section{Discussion}

The results of the EQA showed that 12 of $47(25 \%)$ clinical microbiology laboratories in The Netherlands were not able to identify $C$. auris isolates correctly, at the time that this EQA was conducted. The ability to correctly identify C. auris depended on the manufacturer of the MALDI-TOF, with those laboratories using the Bruker MALDI Biotyper being the most accurate. Only three laboratories that used the bioMérieux Vitek MS system reported a correct identification. However, these laboratories did not use the bioMérieux Vitek MS for identification of $C$. auris to the species level, but used additional identification methods like qPCR, or sequence analysis. The difference between the results of the bioMérieux Vitek MS and the Bruker MALDI Biotyper systems can be explained by the reference databases that were available at the time the EQA was conducted. The CE-IVD approved library version 4.0 of the Bruker MALDI Biotyper IVD system, available in Europe at that time, contained three $C$. auris spectra, while the bioMérieux Vitek MS libraries did not. However, the Knowledge Base V. 3.2 update contains several C. auris spectra, and was CE-IVD marked in June 2018, and is now widely used [27,28].

When the EQA was conducted, several publications reported that the bioMérieux Vitek MS misidentified C. auris isolates as C. lusitaniae, C. haemulonii, or C. albicans, or resulted in no identification, when libraries without $C$. auris spectra were used $[29,30]$. However, when using the RUO database (SARAMIS spectra base) that included C. auris spectra, it should be possible to identify $C$. auris correctly [26]. Of the labs that used bioMérieux Vitek MS, three labs were able to correctly identify C. auris with the methods available in their labs, but only two labs reported that they would perform additional testing when potential misidentifications with Vitek MS were possible (e.g., identification as C. haemulonii, or C. lusitaniae, or no identification). Two other labs specified that they would send isolates from blood cultures or sterile materials to a mycology reference laboratory for identification when bioMérieux Vitek MS was unable to provide a species identification. In the latter scenario, C. auris would be potentially missed when cultured from non-sterile samples. However proper identification is relevant for infection control, as C. auris colonization is an important risk factor for transmission [31,32]. Furthermore, C. auris isolates misidentified as C. haemulonii or C. lusitaniae would have been reported as identified. These results indicate that several laboratories in The Netherlands were not aware of the potential risk of misidentification of $C$. auris with bioMérieux Vitek MS, at the time the EQA was conducted.

An important objective of this EQA was to raise awareness about the potential misidentification of $C$. auris and its ability to cause nosocomial outbreaks, as early recognition is pivotal to limit the unrecognized spread of $C$. auris in the hospital environment $[3,9,31,32]$. As similar to the results of a laboratory survey in Belgium that was conducted during the same period, the results of this EQA indicated that not all laboratories were aware of the potential risks of $C$. auris, nor of the limitations of their yeast identification protocols [28]. We believe that our EQA contributed to increasing awareness, and that together with the updated databases of the MALDI-TOF, unexpected cases of $C$. auris would be identified.

In addition to the possible misidentification with MALDI-TOF MS, several reports indicate that the use of conventional biochemical methods, and the use of bioMérieux Vitek 2 YST ID (Vitek 2) cards, may potentially result in misidentification. The API 20C AUX clinical yeast system has been reported to misidentify C. auris as C. sake, or Rhodotorula glutinis, when the isolate has a negative urease reaction $[24,33,34]$. One participating laboratory reported that they used the API 20C AUX system for yeast identification, but only when the bioMérieux Vitek MS would not be able to identify the yeast species. The Vitek 2 may potentially identify C. auris isolates as C. duobushaemulonii, C. haemulonii, or as 
C. famata $[4,22,29,33-39]$. Several participating laboratories reported the use of the Vitek 2 system, but most would only use it when the MALDI-TOF MS failed to speciate the isolates. Only two labs used the bioMérieux Vitek 2 system as a primary yeast identification method. The updated 8.01 bioMérieux Vitek 2 YST ID system does contain C. auris in its database, and thus may identify C. auris correctly. However, a recent multicenter study showed that only $52 \%$ of 35 C. auris isolates from different clades were correctly identified. Interestingly, differences in the performance among clades were observed. These populations are commonly referred to as the South Asian (I), East Asian (II), African (III), and South American (IV) clades. All isolates from the South American (clade IV) $(n=8)$ were correctly identified, whereas 74\% $(n=13)$ of the South Asian (clade I), and only 7\% $(n=10)$ and $0 \%(n=4)$ of isolates from the African (clade III) and East Asian (clade II) were correctly identified [40]. Thus, regardless of having $C$. auris in its database, this study showed that the identification of some clades of $C$. auris remains problematic, and identification of C. auris and C. duobushaemulonii should trigger additional testing to exclude $C$. auris.

Although not used by the participants in this study, several other methods for identification of yeast are known to misidentify $C$. auris isolates. However, these methods are still used by a significant number of laboratories elsewhere in the world. The misidentifications by other methods have been extensively reviewed elsewhere $[25,41]$.

Whole genome analysis suggests the emergence of five different $C$. auris clades [42,43]. It seems that by using reference databases containing more $C$. auris spectra from different clades, most isolates can be identified correctly with MALDI-TOF MS [44]. Thus, it is likely that with the currently updated Bruker MALDI Biotyper and bioMérieux Vitek MS reference libraries, that contain several C. auris spectra, excellent identification may be achieved. However, a multicenter study with bioMérieux Vitek 2 showed that the performance may vary among C. auris clades for this method [40]. Whether or not the performance of MALDI-TOF MS also varies between the $C$. auris clades has not been studied extensively. With the Bruker MALDI Biotyper and a library that contained only three C. auris spectra, a study showed that all 90 (Indian) isolates could be identified as C. auris (South Asian clade I) [22]. A second study showed that $83.6 \%$ and $98.4 \%$ of 61 isolates could be identified as C. auris, with cutoff scores of $>1.7$ and $<1.7$, respectively (East Asia clade II) [45]. Another study used the Bruker MALDI Biotyper to identify isolates belonging to the African clade III [46]. Isolates form the South American clade IV were reported to be correctly identified with the Bruker MALDI Biotyper [3,47]. However, as it was not the objective to evaluate the performance of the MALDI-TOF MS during the latter three studies, no reference methods for the identification were used, and it is not known how many isolates were misidentified. Using the bioMérieux Vitek MS IVD library version 3.2, 59/61 (96.7\%) of Korean isolates could be correctly identified, with a confidence value of $\geq 75$ (East Asian clade II). In addition, it has been shown that $10 \mathrm{CDC}$ reference strains belonging to four clades could be correctly identified by the bioMérieux Vitek MS and Bruker MALDI Biotyper systems [45]. Information about the performance of the identification methods of the possible 5th clade of $C$. auris is not yet available [43]. As only fragmented information and reports about the use of identification methods without comparison to the gold standard methods is available, a comprehensive analysis of a significant number of isolates from all C. auris clades, that evaluates the performance of the two MALDI-TOF MS systems and several qPCR methods, is warranted.

To aid the rapid identification of $C$. auris, many molecular methods have been developed. They are potentially of use in an outbreak situation, as most are sensitive enough to be used on direct material and do not require a culture, in contrast to MALDI-TOF MS. The published papers that have evaluated the molecular methods to detect and identify C. auris have been reviewed elsewhere [25,48].

To our knowledge, to date only two cases of $C$. auris colonization have been identified in The Netherlands [49]. As these cases were imported from endemic regions, it seems critical that identification methods are used that can accurately identify all C. auris clades, in order not to miss cases. The screening of patients for $C$. auris was recently recommended by an expert panel, indicating that laboratory protocols should include the speciation of colonizing Candida isolates. Identified risk 
groups include patients admitted previously to an ICU in an endemic country, and transfers from hospitals that are known to have $C$. auris. When $C$. auris is detected, an infection prevention control that is measured specifically for $C$. auris should be implemented in a timely manner, to prevent (further) spread [32].

\section{Conclusions}

Several laboratories in The Netherlands were not able to correctly identify C. auris isolates. Only 2/14 laboratories that used methods which could result in the misidentification of $C$. auris mentioned that they would perform additional testing, or send isolates to reference laboratories, to exclude C. auris when needed. However, as 45/47 of participating laboratories have a MALDI-TOF MS system available for the identification of yeast species, and the most used MALDI-TOF MS libraries in The Netherlands currently contain several C. auris spectra, we expect that the ability to identify C. auris in The Netherlands has increased significantly.

Author Contributions: Conceptualization and methodology, J.B.B., H.A.L.v.d.L., I.C.-B., P.E.V., and J.F.M.; formal analysis, J.B.B. and I.C.-B.; writing-original draft preparation, J.B.B.; writing-review and editing J.B.B., H.A.L.v.d.L., I.C.-B., P.E.V., and J.F.M.

Funding: This research received no external funding.

Conflicts of Interest: The authors declare no conflict of interest.

\section{References}

1. Mathur, P.; Hasan, F.; Singh, P.K.; Malhotra, R.; Walia, K.; Chowdhary, A. Five-year profile of candidaemia at an Indian trauma centre: High rates of Candida auris blood stream infections. Mycoses 2018, 61, 674-680. [CrossRef] [PubMed]

2. Rhodes, J.; Fisher, M.C. Global epidemiology of emerging Candida auris. Curr. Opin. Microbiol. 2019, 52, 84-89. [CrossRef] [PubMed]

3. Armstrong, P.A.; Rivera, S.M.; Escandon, P.; Caceres, D.H.; Chow, N.; Stuckey, M.J.; Diaz, J.; Gomez, A.; Velez, N.; Espinosa-Bode, A.; et al. Hospital-Associated Multicenter Outbreak of Emerging Fungus Candida auris, Colombia, 2016. Emerg. Infect. Dis. 2019, 25, 1339-1346. [CrossRef] [PubMed]

4. Chowdhary, A.; Anil Kumar, V.; Sharma, C.; Prakash, A.; Agarwal, K.; Babu, R.; Dinesh, K.R.; Karim, S.; Singh, S.K.; Hagen, F.; et al. Multidrug-resistant endemic clonal strain of Candida auris in India. Eur. J. Clin. Microbiol. Infect. Dis. 2014, 33, 919-926. [CrossRef] [PubMed]

5. Adam, R.D.; Revathi, G.; Okinda, N.; Fontaine, M.; Shah, J.; Kagotho, E.; Castanheira, M.; Pfaller, M.A.; Maina, D. Analysis of Candida auris fungemia at a single facility in Kenya. Int. J. Infect. Dis. 2019, 85, 182-187. [CrossRef]

6. Govender, N.P.; Magobo, R.E.; Mpembe, R.; Mhlanga, M.; Matlapeng, P.; Corcoran, C.; Govind, C.; Lowman, W.; Senekal, M.; Thomas, J. Candida auris in South Africa, 2012-2016. Emerg. Infect. Dis. 2018, 24, 2036-2040. [CrossRef] [PubMed]

7. Escandon, P.; Chow, N.A.; Caceres, D.H.; Gade, L.; Berkow, E.L.; Armstrong, P.; Rivera, S.; Misas, E.; Duarte, C.; Moulton-Meissner, H.; et al. Molecular epidemiology of Candida auris in Colombia reveals a highly related, countrywide colonization with regional patterns in amphotericin b resistance. Clin. Infect. Dis. 2019, 68, 15-21. [CrossRef]

8. Schelenz, S.; Hagen, F.; Rhodes, J.L.; Abdolrasouli, A.; Chowdhary, A.; Hall, A.; Ryan, L.; Shackleton, J.; Trimlett, R.; Meis, J.F.; et al. First hospital outbreak of the globally emerging Candida auris in a European hospital. Antimicrob. Resist. Infect. Control 2016, 5, 35. [CrossRef]

9. Adams, E.; Quinn, M.; Tsay, S.; Poirot, E.; Chaturvedi, S.; Southwick, K.; Greenko, J.; Fernandez, R.; Kallen, A.; Vallabhaneni, S.; et al. Candida auris in Healthcare Facilities, New York, USA, 2013-2017. Emerg. Infect. Dis. 2018, 24, 1816-1824. [CrossRef]

10. Mohsin, J.; Hagen, F.; Al-Balushi, Z.A.M.; de Hoog, G.S.; Chowdhary, A.; Meis, J.F.; Al-Hatmi, A.M.S. The first cases of Candida auris candidaemia in Oman. Mycoses 2017, 60, 569-575. [CrossRef] 
11. Sana, F.; Hussain, W.; Zaman, G.; Satti, L.; Khurshid, U.; Khadim, M.T. Candida auris outbreak report from Pakistan: A success story of infection control in ICUs of a tertiary care hospital. J. Hosp. Infect. 2019, 103, 108-110. [CrossRef] [PubMed]

12. Ruiz-Gaitan, A.; Moret, A.M.; Tasias-Pitarch, M.; Aleixandre-Lopez, A.I.; Martinez-Morel, H.; Calabuig, E.; Salavert-Lleti, M.; Ramirez, P.; Lopez-Hontangas, J.L.; Hagen, F.; et al. An outbreak due to Candida auris with prolonged colonisation and candidaemia in a tertiary care European hospital. Mycoses 2018, 61, 498-505. [CrossRef] [PubMed]

13. Lone, S.A.; Ahmad, A. Candida auris-the growing menace to global health. Mycoses 2019, 62, $620-637$. [CrossRef] [PubMed]

14. Saris, K.; Meis, J.F.; Voss, A. Candida auris. Curr. Opin. Infect. Dis. 2018, 31, 334-340. [CrossRef] [PubMed]

15. Rudramurthy, S.M.; Chakrabarti, A.; Paul, R.A.; Sood, P.; Kaur, H.; Capoor, M.R.; Kindo, A.J.; Marak, R.S.K.; Arora, A.; Sardana, R.; et al. Candida auris candidaemia in Indian ICUs: Analysis of risk factors. J. Antimicrob. Chemother. 2017, 72, 1794-1801. [CrossRef] [PubMed]

16. Chowdhary, A.; Sharma, C.; Meis, J.F. Candida auris: A rapidly emerging cause of hospital-acquired multidrug-resistant fungal infections globally. PLoS Pathog. 2017, 13, e1006290. [CrossRef] [PubMed]

17. Arauz, A.B.; Caceres, D.H.; Santiago, E.; Armstrong, P.; Arosemena, S.; Ramos, C.; Espinosa-Bode, A.; Borace, J.; Hayer, L.; Cedeno, I.; et al. Isolation of Candida auris from 9 patients in Central America: Importance of accurate diagnosis and susceptibility testing. Mycoses 2018, 61, 44-47. [CrossRef] [PubMed]

18. Sherry, L.; Ramage, G.; Kean, R.; Borman, A.; Johnson, E.M.; Richardson, M.D.; Rautemaa-Richardson, R. Biofilm-forming capability of highly virulent, multidrug-resistant Candida auris. Emerg. Infect. Dis. 2017, 23, 328-331. [CrossRef] [PubMed]

19. Kean, R.; Sherry, L.; Townsend, E.; McKloud, E.; Short, B.; Akinbobola, A.; Mackay, W.G.; Williams, C.; Jones, B.L.; Ramage, G. Surface disinfection challenges for Candida auris: An in-vitro study. J. Hosp. Infect. 2018, 98, 433-436. [CrossRef]

20. Short, B.; Brown, J.; Delaney, C.; Sherry, L.; Williams, C.; Ramage, G.; Kean, R. Candida auris exhibits resilient biofilm characteristics in vitro: Implications for environmental persistence. J. Hosp. Infect. 2019, 103, 92-96. [CrossRef]

21. Abdolrasouli, A.; Armstrong-James, D.; Ryan, L.; Schelenz, S. In vitro efficacy of disinfectants utilised for skin decolonisation and environmental decontamination during a hospital outbreak with Candida auris. Mycoses 2017, 60, 758-763. [CrossRef] [PubMed]

22. Kathuria, S.; Singh, P.K.; Sharma, C.; Prakash, A.; Masih, A.; Kumar, A.; Meis, J.F.; Chowdhary, A. Multidrug-resistant Candida auris misidentified as Candida haemulonii: Characterization by Matrix-Assisted Laser Desorption Ionization-Time of Flight Mass Spectrometry and DNA sequencing and its antifungal susceptibility profile variability by Vitek 2, CLSI broth microdilution, and Etest method. J. Clin. Microbiol. 2015, 53, 1823-1830. [CrossRef] [PubMed]

23. Prakash, A.; Sharma, C.; Singh, A.; Kumar Singh, P.; Kumar, A.; Hagen, F.; Govender, N.P.; Colombo, A.L.; Meis, J.F.; Chowdhary, A. Evidence of genotypic diversity among Candida auris isolates by multilocus sequence typing, matrix-assisted laser desorption ionization time-of-flight mass spectrometry and amplified fragment length polymorphism. Clin. Microbiol. Infect. 2016, 22, 277e1-277e9. [CrossRef] [PubMed]

24. Mizusawa, M.; Miller, H.; Green, R.; Lee, R.; Durante, M.; Perkins, R.; Hewitt, C.; Simner, P.J.; Carroll, K.C.; Hayden, R.T.; et al. Can Multidrug-Resistant Candida auris Be Reliably Identified in Clinical Microbiology Laboratories? J. Clin. Microbiol. 2017, 55, 638-640. [CrossRef] [PubMed]

25. Kordalewska, M.; Perlin, D.S. Identification of drug resistant Candida auris. Front. Microbiol. $2019,10$. [CrossRef]

26. Girard, V.; Mailler, S.; Chetry, M.; Vidal, C.; Durand, G.; van Belkum, A.; Colombo, A.L.; Hagen, F.; Meis, J.F.; Chowdhary, A. Identification and typing of the emerging pathogen Candida auris by matrix-assisted laser desorption ionisation time of flight mass spectrometry. Mycoses 2016, 59, 535-538. [CrossRef] [PubMed]

27. Voelker, R. New test identifies Candida auris. JAMA 2018, 319, 2164. [CrossRef] [PubMed]

28. Dewaele, K.; Lagrou, K.; Frans, J.; Hayette, M.P.; Vernelen, K. Hospital laboratory survey for identification of Candida auris in Belgium. J. Fungi 2019, 5, 84. [CrossRef]

29. Wattal, C.; Oberoi, J.K.; Goel, N.; Raveendran, R.; Khanna, S. Matrix-assisted laser desorption ionization time of flight mass spectrometry (MALDI-TOF MS) for rapid identification of micro-organisms in the routine clinical microbiology laboratory. Eur. J. Clin. Microbiol. Infect. Dis. 2017, 36, 807-812. [CrossRef] 
30. Kim, T.H.; Kweon, O.J.; Kim, H.R.; Lee, M.K. Identification of uncommon Candida species using commercial identification systems. J. Microbiol. Biotechnol. 2016, 26, 2206-2213. [CrossRef]

31. Chowdhary, A.; Voss, A.; Meis, J.F. Multidrug-resistant Candida auris: 'New kid on the block' in hospital-associated infections? J. Hosp. Infect. 2016, 94, 209-212. [CrossRef] [PubMed]

32. Kenters, N.; Kiernan, M.; Chowdhary, A.; Denning, D.W.; Peman, J.; Saris, K.; Schelenz, S.; Tartari, E.; Widmer, A.; Meis, J.F.; et al. Control of Candida auris in healthcare institutions. outcome of an ISAC expert meeting. Int. J. Antimicrob. Agents 2019, 54, 400-446. [CrossRef] [PubMed]

33. Lee, W.G.; Shin, J.H.; Uh, Y.; Kang, M.G.; Kim, S.H.; Park, K.H.; Jang, H.C. First three reported cases of nosocomial fungemia caused by Candida auris. J. Clin. Microbiol. 2011, 49, 3139-3142. [CrossRef] [PubMed]

34. Chowdhary, A.; Sharma, C.; Duggal, S.; Agarwal, K.; Prakash, A.; Singh, P.K.; Jain, S.; Kathuria, S.; Randhawa, H.S.; Hagen, F.; et al. New clonal strain of Candida auris, Delhi, India. Emerg. Infect. Dis. 2013, 19, 1670-1673. [CrossRef] [PubMed]

35. Morales-Lopez, S.E.; Parra-Giraldo, C.M.; Ceballos-Garzon, A.; Martinez, H.P.; Rodriguez, G.J.; Alvarez-Moreno, C.A.; Rodriguez, J.Y. Invasive infections with multidrug-resistant yeast Candida auris, Colombia. Emerg. Infect. Dis. 2017, 23, 162-164. [CrossRef] [PubMed]

36. Calvo, B.; Melo, A.S.; Perozo-Mena, A.; Hernandez, M.; Francisco, E.C.; Hagen, F.; Meis, J.F.; Colombo, A.L. First report of Candida auris in America: Clinical and microbiological aspects of 18 episodes of candidemia. J. Infect. 2016, 73, 369-374. [CrossRef] [PubMed]

37. Emara, M.; Ahmad, S.; Khan, Z.; Joseph, L.; Al-Obaid, I.; Purohit, P.; Bafna, R. Candida auris candidemia in Kuwait, 2014. Emerg. Infect. Dis. 2015, 21, 1091-1092. [CrossRef]

38. Ben-Ami, R.; Berman, J.; Novikov, A.; Bash, E.; Shachor-Meyouhas, Y.; Zakin, S.; Maor, Y.; Tarabia, J.; Schechner, V.; Adler, A.; et al. Multidrug-resistant Candida haemulonii and C. auris, Tel Aviv, Israel. Emerg. Infect. Dis. 2017, 23, 195-203. [CrossRef]

39. Kumar, A.; Prakash, A.; Singh, A.; Kumar, H.; Hagen, F.; Meis, J.F.; Chowdhary, A. Candida haemulonii species complex: An emerging species in India and its genetic diversity assessed with multilocus sequence and amplified fragment-length polymorphism analyses. Emerg. Microbes Infect. 2016, 5, e49. [CrossRef]

40. Ambaraghassi, G.; Dufresne, P.J.; Dufresne, S.F.; Vallieres, E.; Munoz, J.F.; Cuomo, C.A.; Berkow, E.L.; Lockhart, S.R.; Luong, M.L. Identification of Candida auris using the updated 8.01 VITEK(R)2 yeast identification system: A multi-laboratory evaluation study. J. Clin. Microbiol. 2019. [CrossRef]

41. Jeffery-Smith, A.; Taori, S.K.; Schelenz, S.; Jeffery, K.; Johnson, E.M.; Borman, A.; Manuel, R.; Brown, C.S. Candida auris: A Review of the Literature. Clin. Microbiol. Rev. 2018, 31. [CrossRef] [PubMed]

42. Lockhart, S.R.; Etienne, K.A.; Vallabhaneni, S.; Farooqi, J.; Chowdhary, A.; Govender, N.P.; Colombo, A.L.; Calvo, B.; Cuomo, C.A.; Desjardins, C.A.; et al. Simultaneous emergence of multidrug-resistant Candida auris on 3 continents confirmed by whole-genome sequencing and epidemiological analyses. Clin. Infect. Dis. 2017, 64, 134-140. [CrossRef] [PubMed]

43. Chow, N.A.; de Groot, T.; Badali, H.; Abastabar, M.; Chiller, T.M.; Meis, J.F. Potential fifth clade of Candida auris, Iran, 2018. Emerg. Infect. Dis. 2019, 25, 1780-1781. [CrossRef] [PubMed]

44. Bao, J.R.; Master, R.N.; Azad, K.N.; Schwab, D.A.; Clark, R.B.; Jones, R.S.; Moore, E.C.; Shier, K.L. Rapid, Accurate Identification of Candida auris by using a novel matrix-assisted laser desorption ionization-time of flight mass spectrometry (MALDI-TOF MS) database (Library). J. Clin. Microbiol. 2018, 56. [CrossRef] [PubMed]

45. Kwon, Y.J.; Shin, J.H.; Byun, S.A.; Choi, M.J.; Won, E.J.; Lee, D.; Lee, S.Y.; Chun, S.; Lee, J.H.; Choi, H.J.; et al. Candida auris clinical isolates from South Korea: Identification, antifungal susceptibility, and genotyping. J. Clin. Microbiol. 2019, 57. [CrossRef] [PubMed]

46. Eyre, D.W.; Sheppard, A.E.; Madder, H.; Moir, I.; Moroney, R.; Quan, T.P.; Griffiths, D.; George, S.; Butcher, L.; Morgan, M.; et al. A Candida auris outbreak and its control in an intensive care setting. N. Engl. J. Med. 2018, 379, 1322-1331. [CrossRef] [PubMed]

47. Parra-Giraldo, C.M.; Valderrama, S.L.; Cortes-Fraile, G.; Garzon, J.R.; Ariza, B.E.; Morio, F.; Linares-Linares, M.Y.; Ceballos-Garzon, A.; de la Hoz, A.; Hernandez, C.; et al. First report of sporadic cases of Candida auris in Colombia. Int. J. Infect. Dis. 2018, 69, 63-67. [CrossRef] 
48. Kordalewska, M.; Perlin, D.S. Molecular diagnostics in the times of surveillance for Candida auris. J. Fungi 2019, 5, 77. [CrossRef]

49. Vogelzang, E.H.; Weersink, A.J.L.; van Mansfeld, R.; Chow, N.A.; Meis, J.F.; van Dijk, K. The first two cases of Candida auris in The Netherlands. J. Fungi 2019, 5, 91. [CrossRef] 